\title{
Le triple assassinat de la rue Montaigne : le sacre
} du fait divers

Prologue

\section{Frédéric Chauvaud}

\section{(2) OpenEdition}

\section{Journals}

Édition électronique

URL : http://journals.openedition.org/abpo/141

DOI : $10.4000 /$ abpo. 141

ISBN : 978-2-7535-1513-0

ISSN : 2108-6443

Éditeur

Presses universitaires de Rennes

Édition imprimée

Date de publication : 1 mars 2009

Pagination : 13-28

ISBN : 978-2-7535-0875-0

ISSN : 0399-0826

Référence électronique

Frédéric Chauvaud, «Le triple assassinat de la rue Montaigne : le sacre du fait divers », Annales de Bretagne et des Pays de l'Ouest [En ligne], 116-1 | 2009, mis en ligne le 31 décembre 2009, consulté le 20 avril 2019. URL : http://journals.openedition.org/abpo/141 ; DOI : 10.4000/abpo.141 


\title{
Prologue \\ Le triple assassinat de la rue Montaigne : le sacre du fait divers
}

\author{
Frédéric CHAUVAUD \\ Professeur d'histoire contemporaine, \\ université de Poitiers, \\ responsable de l'équipe "Sociétés conflictuelles » \\ du GERHICO-CERHILIM
}

En 1887, rue Montaigne, à Paris, trois cadavres de femmes sont retrouvés, égorgées, étendues sur le sol d'un vaste appartement ${ }^{1}$. Cette affaire criminelle, dont on peut faire de multiples lectures, se caractérise d'abord par son extrême violence. De la sorte, elle renseigne sur les seuils de l'admissible et les sensibilités sociales d'une époque. Une demi-mondaine, Madame de Montille, sa fille adoptive et une femme de chambre, native comme sa maîtresse de Chalon-sur-Saône, sont donc trouvées assassinées. Au-delà du crime et de la "Belle Affaire », ce "drame judiciaire » est aussi un révélateur de la place prise par le fait divers. Sans doute, Le triple assassinat de la rue Montaigne est-il aussi important que le «crime de Pantin », commis à la fin du Second Empire et restitué par Michelle Perrot ${ }^{2}$. Sans doute annonce-t-il les récits de crime de la Belle Époque, étudiés par Dominique Kalifa ${ }^{3}$, et scrutés comme un "texte", par Anne Claude Ambroise-Rendu ${ }^{4}$. Mais cette affaire offre un autre intérêt encore : elle incarne un moment particulier et décisif, celui où enquêteurs et journalistes se livrent une lutte incessante pour relever les traces, trouver le coupable et donner aux lecteurs tous les

1. Une première version de cette contribution a été présentée à l'École Nationale de la magistrature.

2. Perrot, Michelle, «L'affaire Troppmann (1869) », L'Histoire, $n^{\circ} 30$, janvier 1981, p. 2837, repris dans Les ombres de I'histoire, Paris, Flammarion, 2001, p. 283-298.

3. Kalifa, Dominique, L'Encre et le sang : récits de crimes et société à la Belle Époque, Paris, Fayard, 1995, 351 p.

4. Ambroise-Rendu, Anne Claude, Petits récits des désordres ordinaires, Paris, Seli Arslan, Paris, 2004, 332 p. 
noms des protagonistes et les éléments de l'intrigue. Si le fait divers est bien un "art de masse ${ }^{5}$ », il devient aussi le terrain d'affrontements parfois vifs dont les enjeux visent au contrôle de l'enquête judiciaire.

En 1887, les recherches policières et judiciaires, après divers tâtonnements et péripéties, s'orientent vers Pranzini qui incarne un monde interlope ${ }^{6}$. À sa manière, il est l'un des représentants de l'Europe des capacités, comme jadis, en 1836, Lacenaire ${ }^{7}$. Entré à 17 ans dans l'administration des postes, il est condamné une première fois en 1877 pour vol de lettres. De la sorte, c'est aussi une carrière criminelle qui est retracée, et l'on sait que la récidive, présentée comme le grand fléau social de la fin du siècle, est assimilée à un mal que l'on ne peut guérir ${ }^{8}$. Celui qui a commis une première fois une infraction ne peut plus s'amender. Les portraits dessinés de Pranzini laissent supposer que son destin est déjà scellé et que seul le malheur se présente à I'horizon. Sa maîtrise des langues - il en aurait parlé cinq couramment - lui donne la possibilité de connaître une singulière existence. II devient l'interprète du général Skobeleff pendant la campagne de Plewa. Ensuite, il voyage à travers l'Afghanistan, le Bélouchistan, la Perse, la Birmanie. Il se fixe en Europe et entre au service de la compagnie Pulmann. Il la quitte précipitamment, accusé de vol. L'affaire fut, semble-t-il, étouffée par la direction par crainte du scandale. Il retourne ensuite en Egypte, est enrôlé dans l'armée anglaise pendant la guerre du Soudan, revient en France en 1886. Le supplément du Grand Dictionnaire universel de Pierre Larousse lui consacre deux colonnes. Pranzini est présenté comme un «aventurier de la pire espèce, né en Egypte de parents italiens ${ }^{9}$ ". Lors du procès, le chroniqueur judicaire le plus célèbre de son temps, Albert Bataille, le décrit le premier jour de l'audience, le 11 juillet 1887 :

"Très calme, très maître de lui, à l'aise sur le banc de la Cour d'assises, il répond avec une politesse onctueuse, tournant avec une rouerie extraordinaire les questions embarrassantes, tantôt se campant devant la Cour, les bras croisés, avec des effets de manchette, l'air content de lui, comme s'il posait pour les belles dames des places réservées; tantôt débitant avec un incroyable aplomb les fables les plus énormes ${ }^{10}$. »

Héros du fait divers et du procès, il est le «tueur de gueuses», le «tueur de courtisanes», voire le "chéri magnifique » qui a enflammé l'imagination

5. BARTHES, Roland, "Structures du fait divers ", Essais, critiques, Paris, Seuil, 1964, p. $188-190$.

6. Chauvaud, Frédéric, "Le «vampire » et le tueur sanguinaire : deux figures de l'étranger au XIXe siècle », dans NourRISSON, Didier, et PERRIN, Yves (dir.), Le Barbare, l'étranger : images de l'autre, Saint-Étienne, Publications de I'Université de Saint-Étienne, p. 215-228.

7. Demartini, Anne Emmanuelle, L'affaire Lacenaire, Paris, Aubier, 2001, 430 p.

8. Briegel, Françoise, et Porret, Michel, (dir.), Le Criminel endurci. Récidivistes du Moyen Âge au xxe siècle, Genève, Droz, 2006, 395 p.

9. LAROUSSE, Pierre, Grand Dictionnaire universel du XIX siècle, tome dix-septième, deuxième supplément, p. 1735-1736.

10. Batallle, Albert, "Pranzini », Causes criminelles et mondaines de 1887-1888, Paris, E. Dentu, 1888, p. 428-429. 
d'une époque et qui sera supplanté, après la Première Guerre mondiale, par d'autres personnages criminels comme Landru.

\section{La mémoire et la rumeur}

L'affaire commence par la découverte des corps étendus des victimes. Les trois cadavres sont presque décapités. La violence inouïe des coups portés a même vivement affecté les policiers arrivés sur place: "Le commissaire de police, ses agents regardaient cette scène avec horreur ${ }^{11}$. » Goron, le souschef de la Sûreté, confie «il m'avait été donné de constater déjà quelques crimes, mais je n'avais jamais vu une boucherie semblable ${ }^{12}$ ». Sur place, Guillot, le juge d'instruction, Gragnon, le préfet de police, et quelques autres restent tétanisés par le spectacle de la violence et du saccage des corps: " cheveux rouges de sang », corps « labouré de blessures profondes ».

La rareté de ce type de crimes a encouragé les études médico-légales et initié une réflexion sur la brutalité, la force et la violence. Les Archives d'Anthropologie criminelle d'Alexandre Lacassagne en rendront compte. Elles représentent alors une sorte d'opinion publique des savants. Brouardel, l'expert diligenté par la Justice, le dira lors de l'enquête : les trois femmes avaient été égorgées avec " une véritable science de boucher ». À l'audience, il trouvera des mots simples pour décrire l'horreur: " elle avait été presque décapitée dans son lit, entre deux oreillers. La tête ne tenait plus au tronc que par un mince lambeau de peau » et d'ajouter : "L'enfant avait essayé de se protéger en entourant sa tête de son bras droit, qui était tailladé de quatre profondes blessures ${ }^{13}$. "Sobre dans le choix des images et des formules, le doyen de la faculté de médecine de Paris signe un compte rendu d'autopsie fait pour éclairer l'enquête et les juges populaires, c'est-à-dire les jurés qui décideront de la culpabilité de l'accusé. En refusant le sensationnalisme et un discours trop technique, il donne le sentiment à tous les membres du jury que ce sont eux qui se forgent une opinion personnelle sans que celleci leur soit dictée par une sommité de la médecine légale. Mais on imagine sans mal la transcription journalistique de la scène, avec ses variantes, ses exagérations et les commentaires qui les accompagnent. Cependant, ce fait divers apparaît bien d'emblée, ainsi que l'affirmait Georges Auclair, comme une "dérogation à la norme ${ }^{14}$ ", ou comme un "fait surprenant ", selon cette fois la formule de Roland Barthes ${ }^{15}$. Dans la presse, le registre de l'émotion domine. II dispense d'explications du contexte, d'analyses du passage à l'acte, d'approfondissements des logiques individuelles et sociales permettant de comprendre.

11. Goron, Les mémoires de M. Goron, Paris, Ernest Flammarion, 1897-1898, t. 2, p. 5.

12. Ibidem, p. 7.

13. Brouardel, Paul, déposition, dans Bataille, Albert, "Pranzinzi », Causes criminelles et mondaines, op. cit., p. 451-452,

14. AuCLAIR, Georges, Le Mana quotidien. Structures et fonctions de la chronique des faits divers, Paris, éditions Anthropos, 1970, 276 p., p. 35.

15. BARTHES, Roland, « Structure du fait divers », art. cit., p. 189. 
C'est l'assassinat de la petite fille qui suscite l'émotion la plus vive. Le Radical, Le Petit National, Le Matin évoquent la sauvagerie du crime. La petite fille avait la tête séparée du tronc, rapportent-ils avec insistance. Dans sa plaidoirie, l'avocat de la défense se demandera en interrogeant les jurés : "Qu'est-ce qui impressionne, en effet, dans cette affaire? C'est la mort de l'enfant! Qu'est-ce qui fait que tous les jours, moi, qui viens remplir ici une mission de justice, moi, qui accomplis un devoir imposé, je reçois des lettres d'injures... Pourquoi ? C'est à cause de l'enfant! » Ainsi, l'opinion publique se manifeste de diverses façons : à l'avocat sont dévolus l'opprobre, l'injure ou le propos haineux; au président des assises sont réservées des félicitations pour son combat contre le "monstre ». Après le verdict et la condamnation à mort, lorsque le pourvoi en cassation est rejeté, il ne reste plus comme seule possibilité, dans le droit français, la grâce présidentielle. Le chef de l'État ne reste pas indifférent à l'émoi collectif qui s'est emparé d'une partie du pays. Il reçoit, écrit-on dans la presse, sans que l'on puisse le vérifier, un grand nombre de lettres, dont les signataires sont principalement des femmes.

Pour prendre la mesure de l'affaire, on peut s'attacher à sa mémoire. Qu'elle soit réminiscence ou construction du passé, elle atteste de l'existence d'une opinion publique qui s'inscrit dans la durée. Plusieurs indices permettent d'en prendre la mesure et d'esquisser une sorte de cheminement. La presse satirique, comme L'Assiette au Beurre ou Gil-Blas, du moins jusqu'à la veille de 1914, fait souvent référence à des criminels célèbres, mais seuls une dizaine d'entre eux jouent les premiers rôles ainsi que l'illustre un dessin humoristique paru dans Le Rire ${ }^{16}$, dans lequel l'artiste, malicieux, a fait figurer son nom, avec celui de Troppmann et de Pranzini (Figure 1).

Dans un tout autre registre, les mémoires de Thérèse Martin, la future sainte Thérèse de l'Enfant-Jésus, qui entra au carmel de Lisieux un an après l'affaire Pranzini, illustrent à la fois la trajectoire d'une existence humaine à I'heure des choix et la notoriété du criminel. Elle écrit en effet: : J'entendis parler d'un grand criminel - du nom de Pranzini - condamné à mort pour des meurtres épouvantables, et dont l'impénitence faisait craindre une éternelle damnation ». Dans l'instant, elle souhaite ardemment que cette dernière ne se réalise pas; aussi s'adresse-t-elle à Dieu : "C'est mon premier pécheur; à cause de cela, je vous demande seulement un signe de repentir pour ma simple consolation. » Le lendemain de l'exécution de Pranzini :

" J'ouvre avec empressement le journal La Croix et que vois-je? Ah! mes larmes trahirent mon émotion et je fus obligée de m'enfuir. Pranzini, sans confession, sans absolution, était monté sur l'échafaud; déjà les bourreaux l'entraînaient vers la fatale bascule quand, remué tout à coup par une inspiration subite, il se retourne, saisit un crucifix que lui présentait le prêtre et baise par trois fois ses "plaies sacrées"».

16. Le Rire, $n^{\circ} 279,10$ mars 1900, dessin de Dépaquit. 


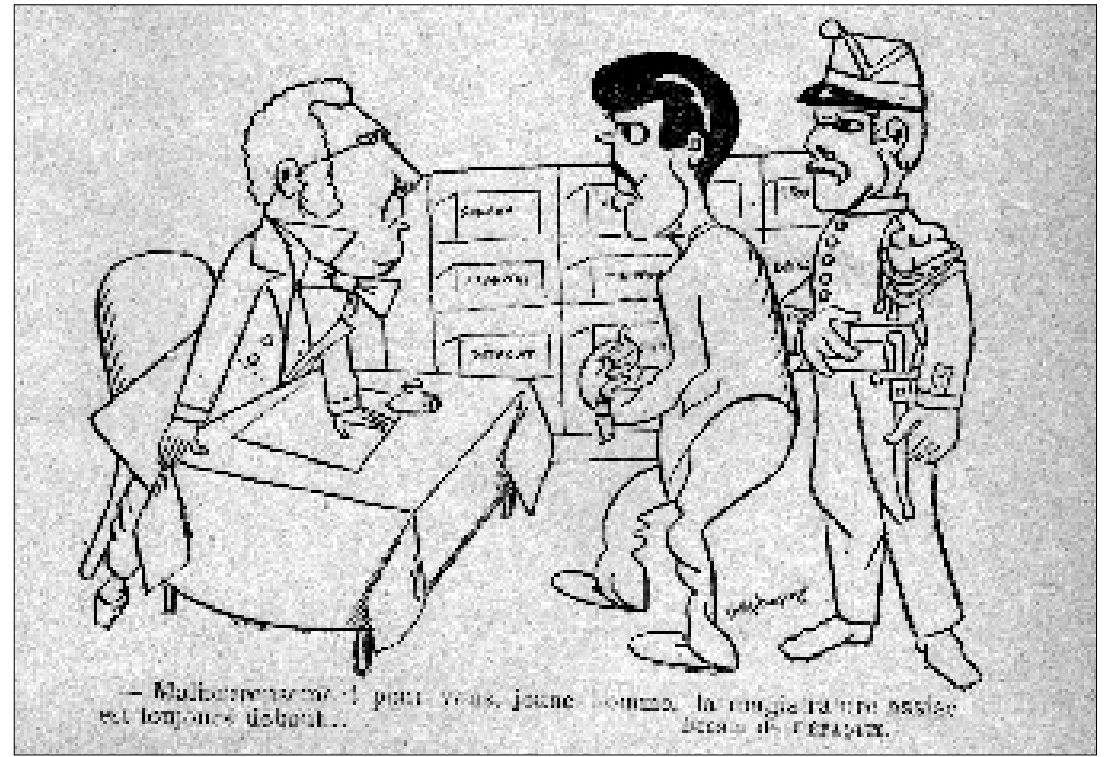

Nul doute que Pranzini ne pensait pas que son dernier geste puisse avoir cette conséquence.

Bien plus tard encore, Edmond Locard, I'inventeur de la criminalistique, relate une anecdote qui se veut amusante et qui confirme la notoriété persistante de l'affaire. Installant à Lyon un musée du crime, il est à la recherche de pièces inédites, éclairantes et si possible célèbres. Un jour, chez un marchand forain, il trouve deux crânes : celui de Pranzini jeune et celui de Pranzini adulte ${ }^{17}$. André Pascal, qui publie en 1933 un Pranzini qui connaîtra vingt éditions, mentionne que, depuis de longues années, " aucun crime n'avait tant ému l'opinion publique que l'affaire de la rue Montaigne ». Rétrospectivement, il affirme que l'intérêt pour l'affaire ira crescendo et qu'elle alimentera toutes les conversations, ajoutant que sur le moment "l'opinion publique fut divisée, comme elle devait l'être, quelques années plus tard, à propos de l'affaire Dreyfus ${ }^{18}$ ". À peu près à la même époque, un magistrat qui fut président de cour d'assises et président de chambre à la Cour de cassation publie aussi une Affaire Pranzini, ainsi qu'une Affaire Lafarge et un Troppmann ${ }^{19}$.

D'autres échos pourraient être mentionnés, mais il importe de s'attacher aussi à l'immédiateté du crime. Que savait-on du crime et de son

17. LOCARD, Edmond, Mémoires d'un criminologiste, Paris, Arthème Fayard, 1957, p. 46.

18. PASCAL, André, Pranzini, Avec reproduction de documents authentiques, Paris, Éditions Émile-Paul Frères, 1933, p. 176.

19. Bouchardon, Pierre, Pranzini, Paris, Albin Michel, 1934, 282 p. 
auteur dans les premières heures? Sans doute, convient-il de distinguer plusieurs strates de l'opinion publique. Parce qu'il s'agit d'un fait exceptionnel, le désordre des lieux, les corps presque étêtés, le mystère qui d'emblée accompagne la découverte de la scène, le crime devient prétexte à toutes sortes de bruissement. La rumeur s'empare très tôt des esprits curieux. Les "premiers bruits qui courent » sont formés par la domesticité, le voisinage et les professionnels de l'information criminelle : "Déjà la nouvelle de l'affreuse tragédie s'était répandue dans Paris, et de tous les côtés arrivaient des gens : concierges, domestiques, locataires de la maison, et, bien entendu, des journalistes ${ }^{20}$. " II ne s'agit pas des grandes rumeurs qui semblent littéralement exploser et annoncent, au gré des révolutions et des grandes secousses politiques, l'arrivée de brigands, la mort du chef de l'État, l'invasion de l'ennemi ${ }^{21}$. La rumeur minuscule, proche du quotidien, négligée par la recherche historique, est pourtant une voie d'accès pour saisir les représentations collectives et les pratiques populaires. La rumeur est un flux instable qui grossit avec le sentiment d'insécurité et le besoin d'information. La rumeur naissante semble d'abord insignifiante. Pour autant, onde presque invisible, elle s'avère irrésistible et semble tout emporter sur son passage. En effet, les voisins de palier, les concierges du 17 rue Montaigne, comme ceux des immeubles voisins, interrogés, se chargent à leur tour de diffuser les "nouvelles», de confronter les récits chimériques et de donner à leurs amis ou à leurs proches des renseignements sur les représentants de l'autorité présents sur place. La rumeur met en scène la trace d'un événement imaginaire ou la transfiguration d'un fait partiellement attesté. Ces rumeurs ne sont que des "cancans de bonnes femmes du quartier » qui peuvent faire perdre du temps aux enquêteurs, voire les dérouter. Ici, elles accréditent l'existence d'un deuxième homme présent sur les lieux ou du moins d'un personnage dont les caractéristiques physiques ne peuvent pas correspondre à celles de Pranzini, mais qui sont à l'origine de «la légende du petit homme brun ${ }^{22}$ ».

La rumeur en circulation s'appuie sur une culture du fait divers qui fonctionne sans doute à la fois par analogie et par sédimentation. Presque aussitôt, en effet, on se rappelle dans les quartiers populaires que d'autres femmes qui facturaient très cher leurs charmes et que l'on ne pouvait donc confondre avec une malheureuse "pierreuse ", victime d'un rôdeur, avaient trouvé la mort depuis 1885 : une première, cité Trévisse, une deuxième rue Condorcet, une troisième rue Bergère, une quatrième rue Caumartin. Le savoir topographique du crime s'enrichit ainsi de connaissances neuves qui donnent aussi au drame une autre dimension : connaître où les crimes ont été commis rend les lieux tangibles et accessibles, puisque l'on peut

20. Pascal, André, Pranzini..., op. cit., p. 45, qui reprend sur ces aspects en grande partie Goron.

21. Voir notamment CoRBIN, Alain, Le Monde retrouvé de Louis-François Pinagot. Sur les traces d'un inconnu, 1798-1876, Paris, Flammarion, 1998, Ch. VIII. «Les invasions », p. 203223.

22. Goron, Les Mémoires, op. cit., t. 2, p. 17. 
se représenter les espaces nommés. L'imagination prend d'autant plus son envol qu'elle s'appuie sur des éléments concrets. Dans l'après-midi, la «loge » de l'immeuble de la rue Montaigne a été envahie par des «gens venus aux informations». Le premier cercle de l'opinion est bien celui de la proximité et des «faiseurs de nouvelles».

Dès le début de la soirée, nombre de journaux donnaient en «Dernière heure » des informations complètes, notamment L'Intransigeant, La Liberté et Le Journal des débats. À la fin du premier jour, la rumeur perd promptement de son importance. Elle est remplacée par le commérage autour des journaux qui donnent, sinon une version officielle, du moins une vision " autorisée » et suffisamment complète pour alimenter de nouvelles supputations, tout en jugulant les bruits les plus fantaisistes qui couraient jusqu'alors. Les interprétations du crime et les interrogations sur son auteur ne peuvent plus se faire que dans le cadre d'un canevas fourni par la presse, faisant office de grille interprétative de ce qu'il faut bien appeler l'événement criminel de l'année. La part de la licence dans le commentaire des faits rapportés se trouve réduite et la clameur publique, faite de mille dialogues singuliers, ressemble à un gigantesque commentaire sur les articles publiés. La construction du fait divers est bien ici un assemblage unissant articles journalistiques et conversations multiples.

\section{Le secret et l'émotion}

Dans l'affaire Pranzini, les services de police les plus prestigieux et les plus efficaces sont distancés par la presse. Entre les deux se mène une course de vitesse qui tourne souvent au désavantage de la police. Goron, sous-chef de la Sûreté, apprend par la presse l'arrestation de Pranzini qui, après avoir quitté Paris, s'était rendu à Marseille. C'est un rédacteur du Journal des débats qui vient le trouver dans son bureau et lui fait lire une dépêche envoyée par leur correspondant marseillais :

«J'étais stupéfait. Ainsi, c'était un journal qui nous apportait la nouvelle de l'arrestation de l'assassin de la rue Montaigne, ou tout au moins d'un complice qui recelait les bijoux volés! Oh! chinoiseries de l'organisation policière! Sainte routine qui fait que la police ignore tous les progrès de la science et que le téléphone et le télégraphe ne profitent qu'aux voleurs, aux assassins... et aux journalistes ${ }^{23} \ldots$ ! »

Pour aller chercher le présumé coupable, soupçonné à ce moment-là d'être un complice, Goron, diligenté par Taylor le chef de la Sûreté, fait lui-même le déplacement. Dans un rapport qu'il adresse à son supérieur hiérarchique, il fait part des relations qu'il entretient avec les médias :

"Inutile de vous dire que les couloirs du palais sont pleins de reporters plus avides de nouvelles les uns que les autres: juges, substituts, commissaires, inspecteurs, garçons de bureaux, sont pressés de questions. Le rédac-

23. Ibidem, p. 47. 
teur du Temps, Guyon, est au même hôtel que moi; je suis aussi discret que possible avec lui, mais il me raconte une partie de ce qui est dit et fait au Palais, où il a des relations ${ }^{24}$. »

Ce constat désabusé, et peut être exagéré, du pouvoir de la presse, suffit en tout cas à montrer l'importance d'un certain journalisme d'investigation ${ }^{25}$ qui dépasse ce que Maurice Talmeyrs évoque dans ces Souvenirs de Journalisme, lui qui avait projeté, avec le dessinateur Forain, des "excursions de police " pour mieux se documenter, en se joignant à des agents de la préfecture pendant leur tournée ${ }^{26}$. Toutefois, les relations sont plus ambiguës qu'elles ne semblent au premier abord. Si la presse est parfois aussi instrumentalisée, plus personne ne doute en revanche de son impact. En effet, tandis que le juge d'instruction marseillais chargé de l'enquête ne parvient pas à trouver la trace d'un témoin qui lui semble capital pour pouvoir reconstituer l'emploi du temps de l'assassin, il fait insérer une note dans plusieurs journaux demandant à cet observateur inconnu de se présenter à son bureau, tout en ayant soin de préciser que le "déménagement » sera payé. L'importance prise par la presse tout comme l'intérêt qu'elle manifeste pour l'affaire Pranzini peuvent être observés visuellement. Le nombre de colonnes concernant l'affaire maintient en haleine un public séduit et captif. Dès le 18 mars, les journaux français et des journaux étrangers consacrent de longs articles à l'affaire. Au bout de quelques jours, les responsables de l'enquête se plaignent que "les feuilles du monde entier » donnent des informations les plus extravagantes, mais aussi les plus précises, comme si les pièces confidentielles réunies lors de l'enquête avaient été directement versées dans les rédactions des journaux. De la sorte, s'ouvre une véritable brèche dans la procédure. En 1887, l'information restait secrète. Les droits de la défense étaient extrêmement limités pendant l'investigation judiciaire et pendant la phase proprement policière de l'enquête. En effet, ce n'est qu'en 1897 que l'avocat a la possibilité d'assister l'inculpé et peut alors intervenir pendant l'interrogatoire. Mais dix ans plus tôt, le déroulement de l'enquête confine au "tapage", car nombre d'aspects sont communiqués à l'extérieur du cénacle judiciaire. Le secret de l'instruction semble totalement chimérique puisque l'on assiste au « déballage des secrets de cabinet sur le théâtre public ». Le représentant de la Sûreté note : " Je dois vous dire qu'ici le procureur de la République fait lui-même des communications à la presse; il est donc bien difficile de garder le secret de quoi que ce soit ${ }^{27}$. " Pratiquement jamais, dans une affaire criminelle, on n'assistera à un tel "scandale ». Ici, il n'est pas question de pièces tombées entre les mains des journalistes, mais bien de renseignements donnés sur l'instruction en cours. La recherche d'informations semble ne

24. Ibid., p. 145.

25. Sur les relations de la presse avec la police, voir notamment KaLIFA, Dominique, L'Encre et le sang, op. cit., p. 200-203.

26. TALmeYr, Maurice, Souvenirs de journalisme, Paris, Plon, 1900, p. 232.

27. ANDRÉ, Pascal, Pranzini..., op. cit., p. 141. 
plus connaître d'obstacles, un peu comme si, au-delà des règles suivies jusqu'alors, tout devenait désormais possible. L'affaire n'étant pas close sur elle-même, elle autorise de multiples rebondissements. De la sorte, le fait criminel n'est pas une simple chronique, il acquiert le statut d'événement, brouillant les frontières avec le fait divers qui peut passionner les lecteurs pendant plusieurs jours, plusieurs semaines, voire plusieurs mois. Un peu plus de sept pour l'affaire Pranzini. Le sens du crime et du procès se vide " au profit de ses virtualités émotionnelles ${ }^{28}$ ». Ce sont elles qui contribuent à rendre translucide le secret de l'instruction. Toutefois, pour aller à la rencontre de l'opinion publique, le fait divers doit s'inscrire dans l'immédiateté et mobiliser l'attention des lecteurs autour de la mort, de la peur et du mystère.

Les descriptions des victimes méritent d'être décryptées. Pour les journalistes, elles donnent aux faits criminels une aura de mystère ou, à défaut, renforcent l'effroi ressenti. D'une façon générale, les victimes intéressent peu la Justice. Souvent même, elles sont suspectées de quelques secrètes turpitudes ou de vices inavouables. Tout se passe en effet, comme si elles avaient, d'une façon ou d'une autre, mérité leur sort ${ }^{29}$. II convient donc tout d'abord de donner aux lecteurs des éléments du décor, autorisant souvent une lecture mi-anthropologique mi-psychologique. En effet, l'intensité de la violence doit transparaître sur les lieux du drame sanglant. À la veille de la Grande Guerre et dans les années trente du xx ${ }^{\mathrm{e}}$ siècle, les spécialistes des indices et des traces alerteront la Justice sur l'importance de tout ce qui peut être recueilli dans l'instant, sur place. En effet tous ces éléments épars, à condition d'être réunis et préservés, peuvent raconter l'épouvante qui pourra être restituée en cour d'assises à l'usage des jurés. Mais la scène du crime, que le journaliste n'a pas nécessairement vue, répond dans les colonnes d'un journal à une autre logique. Imaginée, reconstituée à partir de quelques mots ou, mieux entraperçue, elle doit être donnée sous la forme d'un spectacle sanguinolent: "La jeune femme presque nue, sa chemise de batiste déchirée, gisait sur le tapis, au pied du lit, les bras étendus vers le mur. Le visage baignait dans une large mare de sang. Les traits contractés de la victime, l'expression de terreur de ses grands yeux ${ }^{30} \ldots$ » Peu importe les invraisemblances du récit, il s'agit bien de répondre à une fin : montrer l'horreur en faisant frémir les lecteurs, quitte à ajouter une pointe d'érotisme morbide.

Chaque victime est ainsi l'objet d'un traitement spécifique. Il s'agit d'octroyer à chaque cadavre et chaque mort, donnée par surprise ou reçue en connaissance de cause, ses caractères originaux. II importe ensuite de donner des précisions sur le cadre du crime lui-même : "Sur le tapis du salon, dans une flaque de sang coagulé, apparaissent nettement l'empreinte d'un

28. Nora, Pierre, "Le retour de l'événement ", dans LE Goff, Jacques et Nora, Pierre (dir.), Faire de I'histoire, I. Nouveaux problèmes, Paris, Gallimard, 1974, p. 293.

29. BérARD des Glajeux, Les Passions criminelles, Paris, Plon, 1893, p. 43.

30. GoRon, Les Mémoires, op. cit., p. 37. 
pied d'homme ${ }^{31}$. Le gros orteil était particulièrement bien visible ${ }^{32}$. " Le souci de livrer aux lecteurs des éléments précis et concrets qui fassent vrai l'emporte sur le réalisme. Les descriptions ne sont pas saturées d'explication. En effet, presque personne ne semble se demander pourquoi l'assassin s'est déchaussé. Pourquoi a-t-il marché, après la perpétration de son crime, dans une mare de sang pour y laisser une trace visible? Ces aspects ne sont pas alors l'objet d'interrogations, mais présentés comme des données. Ce qui importe évidemment, c'est de créer une ambiance, de restituer une atmosphère, de donner le sentiment qu'un véritable carnage s'est accompli. L'impression d'ensemble l'emportant sur la crédibilité du détail.

Le récit de la reconstitution du crime, une fois le principal suspect arrêté, constitue à son tour un moment phare de l'affaire. En effet, il ne relève ni tout à fait du fait divers ni de la chronique judiciaire qui s'attache principalement à rendre compte du procès. Lorsque l'assassin présumé est ramené de la capitale phocéenne, il est conduit au parc de Longchamp pour reconstitution. La foule "énorme » devient un personnage à part entière, aussi important que l'auteur du crime. Dès lors, l'opinion publique n'est plus abstraite, elle prend les traits d'hommes et de femmes qui se sont massés sur le trajet ou dans un autre lieu, aux abords de l'appartement des victimes, ou encore non loin de la morgue. L'enterrement des victimes peut à son tour être est mis en intrigue ${ }^{33}$. Faire partager l'émotion collective et le mouvement unanime de compassion devient une sorte d'impératif. Le fait-diversier, plus proche désormais du chroniqueur, tente de se rapprocher du feuilleton ou du « roman larmoyant " très en vogue sous le Second Empire et qui connaît encore au début de la Troisième République, nombre de lecteurs. Dans l'affaire de la rue Montaigne, le convoi funèbre est suivi par des proches, des badauds venus par hasard et un flot de curieux prévenus par la presse. Mais ici, les conditions météorologiques semblent avoir voulu apporter leur concours à la dramatisation du spectacle mortuaire. En effet, "trois catafalques avaient été disposés en avant du chœur. Celui du milieu, drapé de blanc, devait recevoir le cercueil de la pauvre petite Marie ${ }^{34}$ ". L'opinion publique ne se réduit pas, comme lors de la reconstitution, aux lecteurs lointains et anonymes. Malgré les imprécisions sur la date, l'heure et le lieu, I'annonce du report de la cérémonie, la foule " innombrable » est présente. Elle ne s'apparente pas au rassemblement sauvage qui effraie, mais au public policé décrit par Gabriel Tarde. Serrée à l'intérieur de l'église, plus lâche à l'extérieur, la "foule des curieux» est bien, selon les journalistes présents, une "foule considérable», une «foule immense». Il neige sur Paris ce jour-là. De la sorte, sur le trajet qui conduit au cimetière, la neige

31. Voir notamment Beaubatie, Yannick (dir.), Empreintes, Tulle, Mille Sources, 614 p.

32. ANDRÉ, Pascal, Pranzini..., op. cit., p. 43.

33. Riçur, Paul, Temps et récits, Paris, Seuil, t. 1, L'intrigue et le récit historique, p. 66-13 et p. 299.

34. ANDRÉ, Pascal, Pranzini..., op. cit., p. 80. 
blanche recouvre tout. Devenus linceul neigeux, les flocons changent la teinte des autres cercueils et donnent à la scène un caractère fantastique, couvrant d'un voile angélique les silhouettes qui se déplacent sur une surface immaculée pour rendre un dernier hommage aux victimes du crime de la rue Montaigne.

La mise en scène du supplice et le récit du devenir du corps guillotiné des criminels, sur lequel parfois des expériences diverses étaient tentées, constituent aussi un passage obligé du récit de l'exécution capitale ${ }^{35}$. Mais dans l'affaire Pranzini, un éclat particulier vient jeter une lumière sombre sur les pratiques des hommes de l'art diligentés par la Justice. En effet, en 1887, la médecine légale, dont les grands maîtres comme Brouardel, Lacassagne, Vibert et quelques autres accèdent à la notoriété, fait la conquête des prétoires et de la société française. Mais après l'exécution de Pranzini, une fois la tête recueillie et le corps transporté, l'opinion publique, alertée, connaît de vives et inattendues émotions. Le 21 septembre 1887, La Lanterne, feuille radicale fondée dix ans plus tôt et qui se présentait volontiers comme "le journal le plus anticlérical du monde » publie l'article d'un médecin, interne à l'école pratique d'Anatomie, qui fait l'effet d'une bombe. II révèle en effet que sur le cadavre du supplicié, c'est-à-dire de Pranzini, des «fragments tégumentaires » furent prélevés et confiés ensuite à une célèbre maison, située rue de l'école de médecine, pour y être tannés. On en fit deux porte-cartes qui furent offerts aux chef et sous-chef de la Sûreté et un portefeuille que conserva un jeune étudiant. Pendant quatre jours, la campagne se poursuit, suscitant un vif émoi aux allures de scandale tonitruant, mais après tout, semble penser une majorité de lecteurs, il ne s'agissait que du corps d'un criminel. Trouvant un bouc-émissaire en la personne d'un garçon d'amphithéâtre, la campagne de presse finit par retomber pour être très rapidement oubliée, sauf par une petite poignée de spécialistes ${ }^{36}$, dont certains rêvent à la constitution d'un musée crimineloù seraient exposées toutes sortes d'objets.

Lorsque s'ouvre le procès, l'accusé est défendu par maître Demange, I'un des maîtres du barreau. Dans la salle des audiences criminelles, le public de la cour d'assises est un assemblage particulier d'habitués et de curieux occasionnels. La loi prévoit que l'audience est publique, afin que la Justice soit rendue de manière transparente. Dans les faits, les places disponibles étaient peu nombreuses et parfois les présidents d'assises

35. Voir Porret Michel (dir.), Le Corps violenté. Du geste à la parole, Genève, Droz, 1998, 370 p., et pour une perception plus contemporaine, BADINTER, Robert, L'Abolition, Paris, Fayard, 2000, $327 \mathrm{p}$.

36. Notamment Edmond Locard qui donne une version plus personnelle «À la dissection, on préleva sa peau [...] pour en faire des portefeuilles que se partagèrent quelques-uns des chefs de la police, dont les femmes n'eurent rien de plus pressé que de les accaparer et les montrer à tout venant. Cela fit un scandale épouvantable et le préfet de police dut donner sa démission ", LOCARD, Edmond, Mémoire d'un criminologiste, op. cit., p. 46. et le célèbre spécialiste lyonnais d'ajouter «J'ai d'ailleurs eu entre les mains des portefeuilles en peau humaine... ». 
distribuaient des cartes d'invitation réservées à une élite appartenant au monde élégant. En attendant l'entrée de Pranzini et de la cour, il règne en effet dans la salle "une atmosphère de répétition générale ». Des observateurs dénoncent le luxe tapageur qui s'affiche en ces lieux, un luxe qui a sa place "à Longchamp", mais ne convient pas pour la cour d'assises. L'éclat des bijoux, les rires à peine contenus, les collations substantielles apportées avec soi, les éventails bariolés qui s'agitent, tout concourt à donner du public une description où les femmes dominent. Ces dernières se réjouissent, écrivent les chroniqueurs, d'un certain apparat, de la pompe déployée et de l'ostentation du cérémonial judiciaire. Comme pour mieux le souligner, quelques journalistes insistent sur le trouble des cœurs sensibles et pervers de certaines «femmes de bonne éducation » qui ne parviennent plus à quitter des yeux l'assassin, totalement captivées par lui. D'ailleurs, lorsque la "profondeur langoureuse de son regard » est décrite, nul ne s'étonne de l'attraction exercée par Pranzini, aussi bien auprès des « alouettes sans expérience », que des femmes «à la sensibilité déréglée ${ }^{37}$ ».

\section{La dictature du neuf}

Pour entretenir le suspens, faire participer les lecteurs et tenir en haleine l'opinion publique, il existe plusieurs stratégies, dont quelquesunes seulement sont présentées ci-dessous. L'une d'entre elles tient au ton employé, au sentiment de trépidation ou d'urgence que l'article peut contenir. Il s'agit parfois de donner l'impression de la simultanéité, entre le récit et l'action racontée, un peu comme si la scène était transcrite en direct, laissant ouvert l'instant d'après, encore totalement inconnu, au moment où on écrit, alors qu'une chronique qui restitue des événements passés peut être vivante et alerte, mais ne possède pas la même intensité. De la sorte, le recours à l'isochronie s'avère particulièrement efficace. Il s'agit d'un mode de focalisation du récit qui doit fondre le temps du récit, celui de l'histoire et celui de la lecture. Il donne au lecteur le sentiment qu'il est directement associé à l'événement, que son opinion ne se distingue pas de celui du journaliste, et que tous les deux participent, ensemble, à la confection de l'information. Toutefois, le procédé ne peut être utilisé en permanence. II relève de l'artifice et guide plus sûrement le lecteur en ne lui laissant aucune part d'initiative. Pour y parvenir, la rédaction peut choisir de faire figurer des documents "authentiques", en insérant par exemple un télégramme, à la manière de La Dépêche qui en imprime un, en fac-similé. II est également possible de ne recourir qu'à des techniques d'écriture en rapportant entre guillemets des propos: "C'est lui! je le reconnais ${ }^{38}$ ! " Il s'agit ici du cri de la préposée aux toilettes publiques dans lesquelles Pranzini aurait laissé une partie de son «butin ». Le lecteur peut alors avoir

37. Voir notamment, CHAUVAUD, Frédéric, "Les élégantes de la cour d'assises au XIXe siècle », dans BARD, Christine (dir.), Le Genre des territoires, féminin, masculin, neutre, Angers, Presses de l'Université d'Angers, 2004, p. 63-76.

38. PASCAL, André, Pranzini..., op. cit., p. 107. 
le sentiment que le journaliste n'est pas omniscient, mais qu'il livre, dans l'instant, ce qu'il a vu et entendu. Si l'article est rédigé en adoptant un style saccadé, il donne un effet de vérité et d'urgence, renforce les liens entre le journaliste et ses lecteurs, devenus à la fois confidents et complices de l'enquête journalistique.

Entretenir l'intérêt, faire en sorte que le souffle trouvé au début de l'affaire se poursuive sans relâchement, devient une exigence. Mais chaque jour, il faut trouver du nouveau. Dans cette perspective, l'image peut, parfois, jouer un rôle non négligeable. La photographie en particulier, même si elle est figée et proche de portraits officiels, ajoute un élément supplémentaire qui participe de la véracité journalistique. Mieux que la gravure sur bois des canards, assez proche de l'image d'Epinal ou du croquis d'audience, elle n'est pas cantonnée à l'information des jurés d'assises. La photographie offre à chacun un miroir. Elle permet l'identification ou la comparaison. Elle donne aussi l'impression d'apporter une preuve. L'image, semblent dire les contemporains, ne peut mentir. La photographie de Pranzini, réalisée par la maison Chamberlin, est reproduite dans plusieurs journaux, dont Le Monde illustré. Le même périodique offre aux lecteurs les portraits de Goron et de Taylor (Figure 2).

Figure 2



M. Taylor

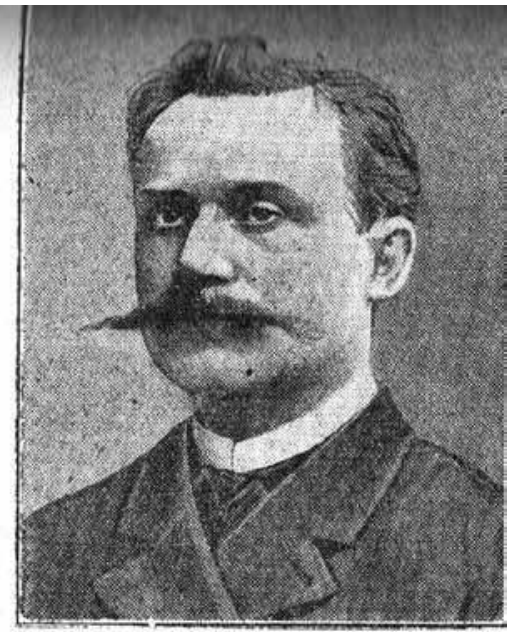

M. Goron

Une autre stratégie consiste à ouvrir au sein du journal un espace baptisé le plus souvent "Courrier des lecteurs » où chacun peut faire part de ses réactions, de son indignation, de sa stupéfaction, de ses doutes. Mais d'autres possibilités existent. Caliban, journaliste au Figaro, organise pour les colonnes de son journal une enquête auprès du lectorat. II s'agit d'une 
sorte de referendum destiné d'abord aux lectrices. II porte sur la conduite de la maîtresse de Pranzini. Cette dernière avait fait des aveux. Elle s'était confiée sans retenue au juge d'instruction. Sa déposition ne reste pas confidentielle bien longtemps et figure intégralement dans les pages de nombre de journaux. Aussi le Figaro pose la question de savoir si elle a eu raison de dire la vérité. Les réponses se révèlent majoritairement négatives. Une majorité de lecteurs et de lectrices prétendent en effet qu'« une amante véritable aurait dû se faire condamner, plutôt que de livrer l'homme qu'elle aimait ${ }^{39}$ ».

La dernière stratégie, la plus importante, consiste, pour la presse, non pas à concurrencer la police, mais à la précéder. De la sorte, il s'agit de devancer l'information ou, plus exactement, d'en être à l'origine. Ainsi, le sous-chef de la Sûreté se rend en Belgique, puis en Allemagne. Deux journaux, Le Temps et Le Soleil, organisent la filature. À Breslau, leurs correspondants occupent les chambres contiguës à celle du policier. Le 21 avril, dans sa troisième livraison, celle de six heures du matin, Le Soleil publie l'article de son rédacteur, daté de la veille au soir. Il offre aux lecteurs des renseignements confidentiels sur le développement de l'enquête et les faits et gestes du «limier » français. Dans la course à l'information et à la primeur des nouvelles, le journal précède ainsi les services de police. Si le sous-chef a bien envoyé un télégramme urgent, quelques heures avant la dépêche du journaliste, cette missive n'a été distribuée que tardivement et n'est parvenue que fort tard au Palais de Justice. Ainsi, le procureur et le juge d'instruction apprennent «la nouvelle » non pas en consultant le message officiel, mais en lisant la presse.

Pour nombre d'observateurs, les années $1885-1889$ sont des années particulières, où chacun fourbit ses armes et au cours desquelles se déroulent des luttes épiques. Les trois « $\mathrm{P}$ » - la police, le parquet et la presse - s'empoignent par le biais de déclarations plus ou moins publiques et de pratiques offensives liées à l'investigation judiciaire. C'est aussi une époque où de grands journaux, à l'instar du Figaro, de La Liberté, de L'Intransigeant, organisent une sorte de "police journalistique » extrêmement dispendieuse pour suivre l'affaire, avant donc que soit dénoncée, de manière plus virulente, "l'indignité du reportage ${ }^{40}$ " portant préjudice, au début du $\mathrm{xx}^{\mathrm{e}}$ siècle, à la bonne marche de la Justice. Les articles consacrés par le seul Figaro au crime de la rue Montaigne, pendant six mois représentent un volume de 400 pages. Une affaire, même extraordinaire, lasse d'ordinaire plus rapidement. Le déroulement de l'instruction, les enquêtes menées par les grands journaux qui se répondent, se concurrencent, contribuent à « empoigner le public ». Si l'année 1887 connaît en décembre le scandale des décorations, la mémoire des contemporains a davantage retenu l'affaire Pranzini qui

39. Ibidem, p. 175.

40. DelPORTE, Christian, Histoire du journalisme et des journalistes en France, Paris, PUF, 1995, p. 31-32. 
est bien le révélateur des transformations structurelles du journalisme ${ }^{41}$, du goût du public, de la diversité des rubriques, des changements techniques et de l'avènement d'une littérature des démocraties modernes ", pour reprendre une formule plus tardive de Zola. 1887, c'est aussi l'année où Fortuné de Boigobey publie La Chambre rouge qui met en scène, pratiquement pour la première fois, une des situations type du roman policier. Dans l'affaire Pranzini, nombreux sont ceux qui ont tenté de multiplier les références littéraires. La scène du crime elle-même semble se mettre au service du romanesque : "Un petit chiffonnier supportait une lampe et plusieurs volumes. Il y en avait même un qui était resté ouvert : c'était un roman intitulé Le Joueur, un récit mélodramatique de l'assassinat d'une fille par son amant de cœur, qui l'avait tuée pour lui voler 2500 francs $^{42}$. » Lors du procès, Albert Bataille, le célèbre chroniqueur du Figaro le souligne à sa manière : "Il y a dans l'affaire Lerouge [1864]; le roman le plus célèbre de feu Gaboriau, un mot qui a dû frapper l'assassin de la rue Montaigne et qui résume tout son système de défense ${ }^{43}$. " À divers stades de l'enquête judiciaire et du procès, nombre de journalistes, à travers un fait extraordinaire, tentent de jeter des ponts avec telle ou telle œuvre romanesque, mêlant ainsi culture feuilletonesque et culture judiciaire pour les fondre dans un vaste creuset qui voit l'affirmation de la culture de masse ${ }^{44}$, à une époque où la lecture se généralise ${ }^{45}$, élargissant considérablement le lectorat, fixant des représentations sociales et culturelles de l'univers du crime, construites à partir de l'écrit. Nul doute que l'affaire Pranzini participe à la construction d'une profession ${ }^{46}$, à l'invention du journalisme ${ }^{47}$ autour de faits divers et à la naissance de la culture médiatique ${ }^{48}$.

41. Voir notamment Charle, Christophe, Le Siècle de la presse, Paris, Seuil, coll. "L'univers historique », 2004, $409 \mathrm{p}$.

42. GORON, Les Mémoires..., op. cit., t. 2, p. 15.

43. BATAlLLE, Albert, Causes célèbres et mondaines, op. cit., p. 428.

44. KALIFA, Dominique, La Culture de masse en France, 1. 1860-1930, Paris, La Découverte, $2001,123 \mathrm{p}$.

45. MolLIER, Jean-Yves, La Lecture et ses publics à l'époque contemporaine. Essais d'histoire culturelle, Paris, PUF, coll. « Le nœud gordien », 2001, 186 p.

46. DeLPORTE, Christian, Les Journalistes en France, 1880-1950. Naissance et construction d'une profession, Paris, Seuil, 1999, $444 \mathrm{p}$.

47. FerenCZI, Thomas, L'Invention du journalisme en France à la fin du xixe siècle, Paris, Payot, 1996, $279 \mathrm{p}$.

48. MolLIER, Jean-Yves, "La naissance de la culture médiatique à la Belle Époque », Études littéraires, 30, 1, 1997. 
RÉSUMÉ

En 1887, un crime sanglant devient le révélateur de la place prise par les faits-divers. L'affaire Pranzini, surnommé le «chéri magnifique » ou le «tueur de courtisanes», a enflammé l'imagination d'une époque. Elle s'avère aussi importante que l'affaire Troppmann pour comprendre comment la «Belle Affaire " est devenue un "art de masse ". Le Grand Dictionnaire universel de Pierre Larousse lui consacre un article, attestant de son importance. Aussi, dans la présente contribution, il s'agit de suivre la "mémoire » de l'affaire, puis de scruter de quelle manière les acteurs de la justice et ceux du journalisme se livrent à une concurrence exacerbée, tout en s'attachant aux techniques de la "fabrique » d'un fait divers devenu fait de société. L'affaire de la rue Montaigne concourt en effet à fixer les représentations sociales et culturelles de l'univers du crime, construites à partir de l'écrit. À différentes étapes de l'enquête, l'émotion, le secret et le scandale alimentent l'angoisse et la curiosité des lecteurs et du public de la cour d'assises dans un récit mélodramatique "de l'assassinat d'une fille par son amant de cœur ». Nul doute que l'affaire Pranzini participe à la construction d'une profession, à l'invention du journalisme autour de fait-divers et à la naissance de la culture médiatique.

\section{ABSTRACT}

In 1887, a bloody crime reveals the space occupied by the "faits divers". The Pranzini affair, called "cheri magnifique" (splendid darling) or "tueur de courtisanes" (the killer of courtesans), has fired the society imagination in those days. It is as important as the Troppmann affair to understand how the "Belle Affaire" became a "art de masse". The Pierre Larousse's Grand dictionnaire universel devotes an article to it, attesting of its importance. This article is aiming at following "the memory" of the affair, then to examine how the parties involved in justice and those involved in journalism devote themselves to an exacerbated competition and how and by which means a "faits divers" become a part of the history society. The affair of the Montaigne Street contributes to set the social and cultural representations of the crime universe, built with the writing. On various stages of the investigation, the emotion, the secret, and the scandal feed the fear and the curiosity of the readers as for the public of "la Cour d'Assises". There is no doubt that the Pranzini affair takes part in the construction of a profession, in the invention of journalism dealing with "fairs divers" and in the birth of media culture. 
Première partie

\section{Le fait divers dans tous ses états}


